\title{
Galaxies at high redshift: progress and prospects
}

\section{Charles C. Steidel}

Charles C. Steidel, "Galaxies at high redshift: progress and prospects," Proc. SPIE 4005, Discoveries and Research Prospects from 8- to 10-Meter-Class Telescopes, (29 June 2000); doi: 10.1117/12.390153 


\title{
Galaxies at high redshift: progress and prospects
}

\author{
Charles C. Steidel ${ }^{a}$ \\ ${ }^{a}$ Palomar Observatory, California Institute of Technology, \\ MS 105-24, Pasadena, CA 91125, USA
}

\begin{abstract}
There has been considerable progress made in the discovery, observation, and understanding of high redshift galaxies in the last few years; most of this progress is attributable to greatly improved spectroscopic throughput made possible by state-of-the-art instruments on the new generation of $8-10 \mathrm{~m}$ telescopes. Here we review a few of the areas in which substantial progress has been made, and discuss the future of high redshift galaxy work in the context of the observational facilities that are either in operation or soon to come.
\end{abstract}

Keywords: galaxy formation, galaxy evolution, cosmology

\section{INTRODUCTION}

It is probably fair to say that the advent of the Keck telescopes, and in particular the commissioning of Low Resolution Imaging Spectrograph ${ }^{1}$ in the latter half of 1994, represented the leading edge of a wave that has revolutionized the study of faint galaxies in the last 5 years. The observational situation has evolved from one in which (normal) galaxies with spectroscopic redshifts in excess of 1 were novelties, and extremely hard-fought, to one where galaxies can be found almost at will to redshifts $z \sim 6$ and where several hundred $z \sim 1$ redshifts can be obtained in a single night of observing. This revolution is currently accelerating rapidly as the VLT UTs, Subaru, and Gemini $8 \mathrm{~m}$ telescopes are coming on line, with their own suite of state-of-the-art instruments.

The gains over $4 \mathrm{~m}$-class telescopes have been unexpected, in many ways. The new $8-10 \mathrm{~m}$ telescopes not only represent substantial gains in collecting area, but they have also been built at the finest terrestrial sites with the best atmospheric stability, and they take advantage of the state of the art in instrumental throughput (e.g., in the case of LRIS, achieving total throughput including telescope and detector of 35-40\%). Whereas many projections in the 1980's and early 1990's predicted the real breakthroughs would be occurring in the infrared (the less-explored discovery space, the rapidly-advancing detector technology coupled with telescopes optimized for IR performance), many of the advances made to date have been based on "traditional" optical spectroscopy. From my own experience, I would estimate that optical spectroscopy with Keck/LRIS represents and approximate 20-fold gain over anything that was feasible with $4 \mathrm{~m}$-class telescopes. The "next-generation" optical spectrographs, such as DEIMOS on Keck II, VIMOS on the VLT, and IMACS on the Magellan $6.5 \mathrm{~m}$ promise even larger gains, mainly through larger field sizes that will allow taking further advantage of multiplexing to significantly improve survey efficiency.

We are also in the midst of an era in which the first sensitive near-IR spectrographs are coming on line- several were commissioned in 1999, including NIRSPEC ${ }^{2}$ on Keck II, ISAAC on VLT UT1, and IRCS on Subaru. The value of (ground-based) near-IR spectroscopy for studies of high redshift galaxies ranges from "essential" in the case of heavily reddened objects or early-type galaxies beyond $z \sim 1$, to "intriguing" for providing access to rest-frame optical wavelengths and the same diagnostic nebular emission lines that have been employed in observations of local galaxies. The ability to detect these lines in the near-IR is expected to help in obtaining redshifts for particular ranges that suffer from a dearth of spectral features falling in the optical window of 0.4-1 micron. There are currently under development a number of near-IR multi-object spectrographs for 8m-class telescopes (e.g., NIRMOS for the VLT, FLAMINGOS, GMOS-IR for Gemini, DEIMOS-IR for Keck). It is difficult to predict what impact these instruments will have on studies of high redshift galaxies; some thoughts on the subject are given below.

In this short contribution, I will review a small subset of what has been accomplished on the universe of high redshift galaxies using $8-10 \mathrm{~m}$ telescopes, followed by a discussion of some of the directions in which future observations might lead. While a very substantial amount of important work has been done on the universe at $z \leq 1 \mathrm{using} 8$-10m telescopes, notably obtaining spectra of unprecedented detail that opens up new astrophysical possibilities ${ }^{3}$ (some

E-mail: ccs@astro.caltech.edu 
of which are discussed in these sessions), I am assuming that my assigned task is to discuss the $z>1$ universe. In what follows, it is this redshift regime, beyond $z \sim 1$, that is meant by the term "high redshift". In addition, the study of high redshift AGN (QSOs and radio galaxies) is a very rich area where a great deal of progress was made prior to the advent of $8-10 \mathrm{~m}$ telescopes; I will not discuss these here, hoping that they will be covered elsewhere in this volume.

\section{OBSERVING THE "EPOCH OF GALAXY FORMATION"}

\subsection{Optical Surveys for High Redshift Galaxies}

The approach to faint galaxy redshift surveys has necessarily evolved, or at least has become more diverse, in order to begin to take in significant numbers of galaxies beyond $z \sim 1$. The problem is that the median redshift of a survey of faint galaxies increases only very slowly with limiting magnitude. For example, the $\mathrm{CFRS}^{4}$ redshift survey, which was apparent magnitude limited $I_{A B}=22.5$, was a truly heroic effort on a $4 \mathrm{~m}$-class telescope. Of $\sim 600$ spectroscopic redshifts, only about $7 \%$ lie at $z \geq 1$; similarly, even with significantly fainter apparent magnitude limits, the first faint galaxy surveys conducted using Keck/LRIS ${ }^{5,6}$ yielded only handfuls of $z>1$ galaxies from samples containing many hundreds of galaxies. There are two possible approaches to compiling samples large enough to study the statistical properties of the galaxy populations even beyond $z \sim 1$ : the first is simply to obtain overwhelmingly large numbers of galaxy redshifts, so that even the small fraction of high $z$ galaxies will yield large numbers of them. This appears to be the approach that will be used by the VIRMOS consortium for their planned major survey efforts. A different approach, which has the disadvantages of added complexity, a need for deeper initial images for target selection, and more difficult post-facto analysis to understand completeness, is to "target" particular redshift ranges using photometric pre-selection. ${ }^{7}$ One advantage to this kind of approach is that it is anywhere from 10-25 times more efficient for accumulating data in a particular constrained cosmological volume (rather than a pencil beam which slices through all redshifts and for which the selection criterion imposed by an apparent magnitude cut in some passband is different at each redshift). Another advantage is that having some idea about the redshifts that are likely to be obtained allows one to optimize the observational setup for success (e.g., choosing the wavelength range covered, the blaze of the grating or grism, the spectral resolution needed to balance information content and efficiency, etc.) Finally, the samples that one can construct using photometric pre-selection are nearly volume-limited, meaning that one can include a significant range in apparent ( $\sim$ absolute) magnitude at a given redshift, enabling much more uniform sampling of the luminosity function and more intrinsically fainter galaxies without paying a price in overall survey efficiency.

Some examples of photometrically-tuned redshift surveys are surveys for "Lyman break galaxies" (LBGs), based on the guaranteed spectral feature at $912 \AA$ in the galaxy rest frame ${ }^{8-11}$ produced by a combination of stellar spectral energy distributions, H I opacity of the galaxy itself to its own Lyman continuum radiation, and H I in the intergalactic medium. The Lyman break enters the ground-based window for $z \gtrsim 2.5$, and broad band filter systems and accompanying color criteria can be tailored to particular redshift ranges (figure 1). The Lyman break method is particularly effective for redshifts $2.5 \lesssim z \lesssim 3.5$ because this places all of the spectral features that are most useful for obtaining redshifts (see $\S 3.1$ below) in the observed wavelength range where optical imaging spectrographs have the highest throughput and where the night sky background is the darkest. A final advantage of photometrically selected redshift surveys is that the full photometric sample, often much larger than the subset with spectroscopic redshifts, can be used (with the knowledge of the selection function from the spectroscopic sample) for many statistical measurements, from angular correlation functions to luminosity distributions. ${ }^{11,12}$

The unprecedentedly large samples that can be assembled with a relatively modest amount of telescope time (obviously the efficiency will be improved by future instruments having larger fields of view) allow for analyses that were somewhat unexpected. First, it is now possible to measure the clustering properties of relatively "normal" star forming galaxies at very high redshift. The clustering properties have arguably told us more about the nature of the high redshift galaxies than any other information, and provide the strongest link to theory at this point in time. The upshot is that high redshift galaxies are very strongly clustered, nearly as strongly clustered as bright galaxies in the present-day universe (Figure 2). This fact has generally been interpreted as providing strong support for hierarchical structure formation models in which early galaxies form within the most massive dark matter halos at high redshift, whose spatial distribution should be strongly biased with respect to the overall mass distribution. ${ }^{14-17}$ Thus, a characteristic mass scale for LBGs comes from an analysis of their clustering properties; for currently-fashionable cosmological parameters of $\Omega_{0}=0.3, \Omega_{\Lambda}=0.7$, this mass scale is $\sim 10^{12} \mathrm{M}_{\odot}$. If one were to follow the LBGs 

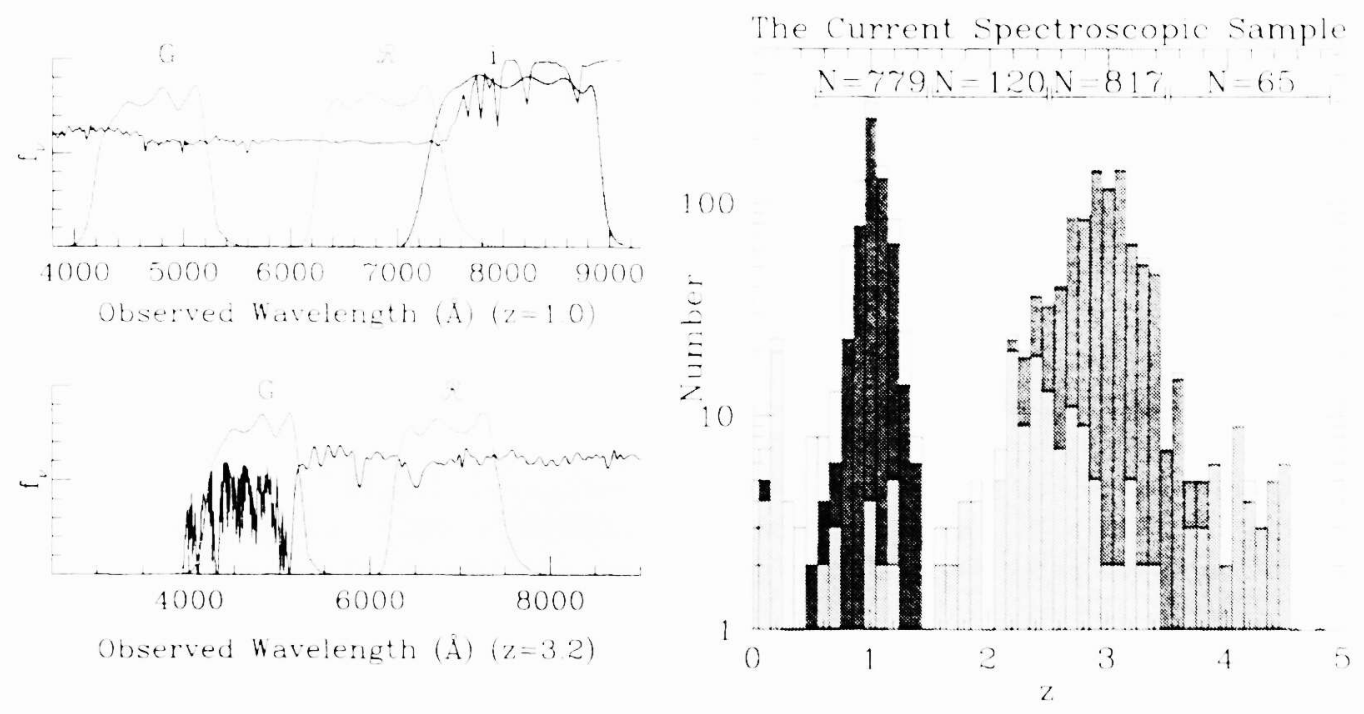

Figure 1. (Left) Crude illustration showing how significant spectral features can be used with a particular set of broad band filters to select galaxies in the vicinity of prescribed redshifts. The top panel illustrates how we can select star forming galaxies at $z \sim 1$ based on the spectral curvature in the vicinity of the Balmer break, while the bottom panel shows the even more pronounced Lyman break near $z \sim 3$. (Right) Redshift histograms from various photometrically-selected samples (different sample selection criteria are coded by different shades of gray). Spectroscopic samples at particular cosmic epochs can be constructed efficiently and quickly using such "targeted" surveys. The sample at $z=1.02 \pm 0.12$, for example ( $\sim 800$ redshifts $)$, is based on only $\sim 4$ nights of spectroscopic effort using Keck/LRIS. ${ }^{13}$
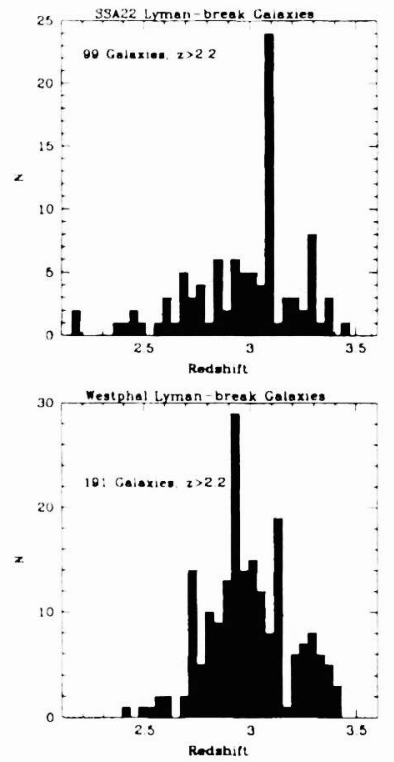
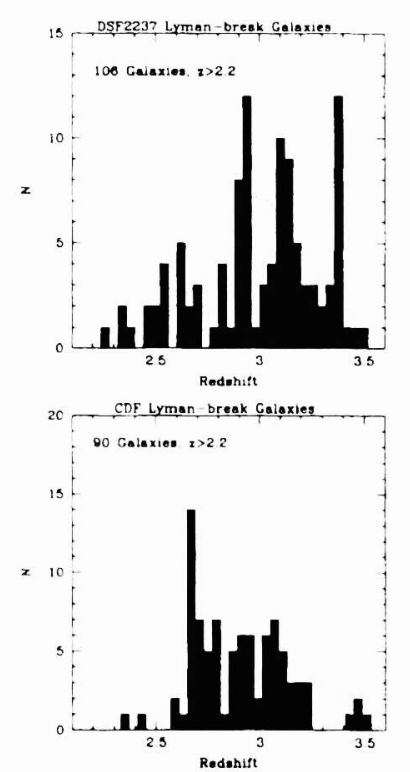

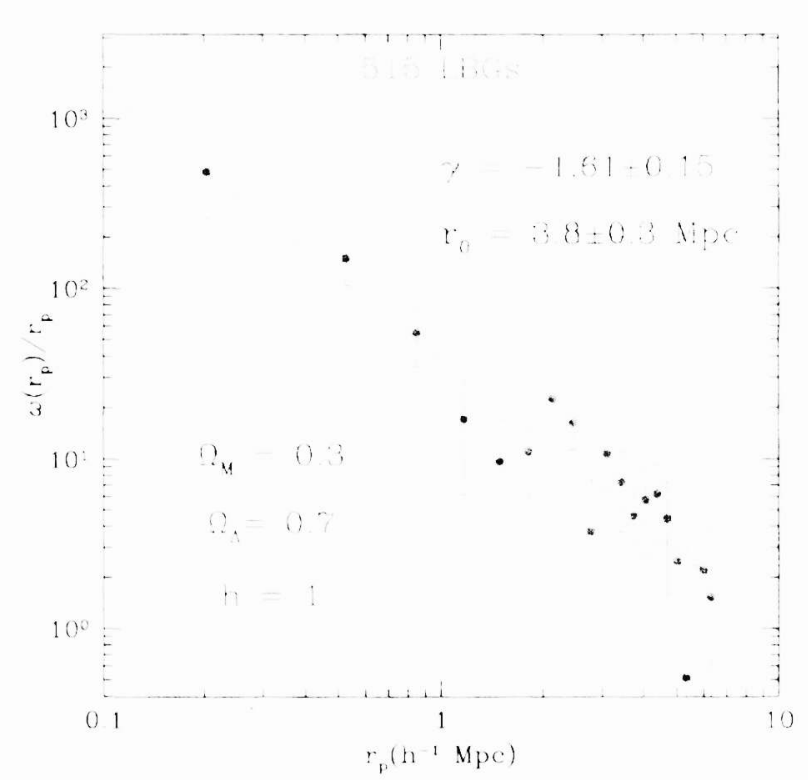

Figure 2. (Left) Redshift histograms of $z \sim 3$ Lyman break galaxies in each of 4 survey fields. The most significant "spikes" are likely to be the progenitors of present-day rich clusters of galaxies. (Right) The preliminary correlation function of bright Lyman break galaxies at $z \sim 3,{ }^{13}$ from a subset of the spectroscopic survey. 

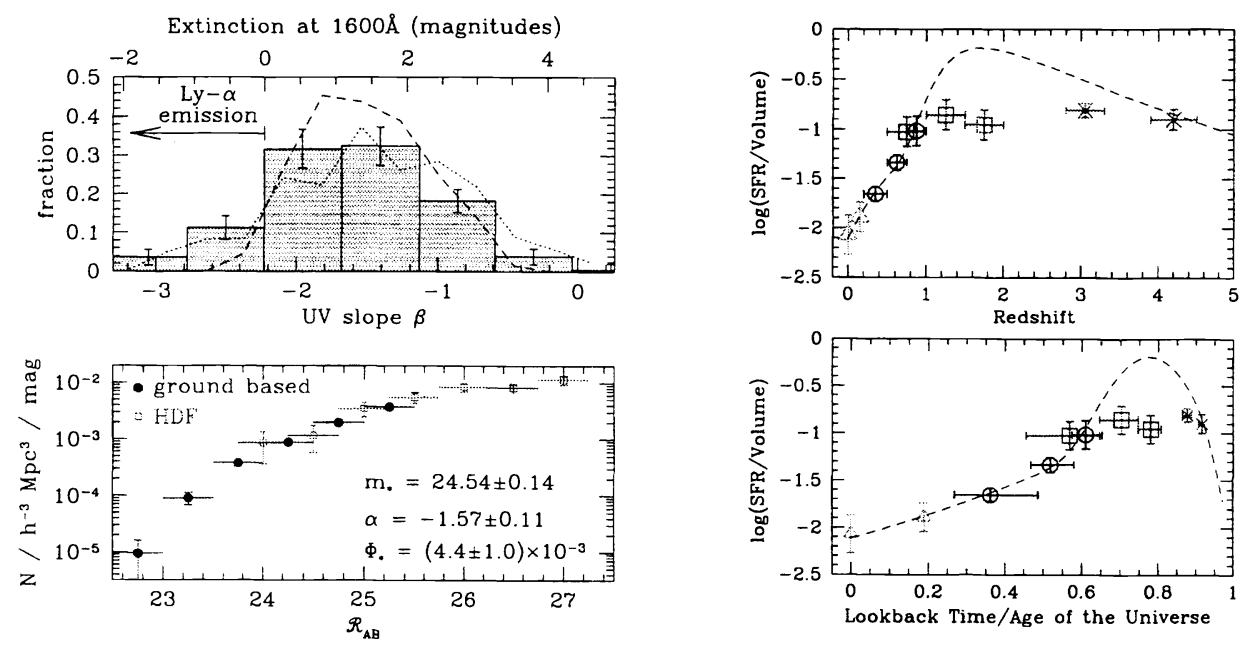

Figure 3. (Left) The top panel shows the inferred distribution of UV extinction for $z \sim 3$ LBGs, while the bottom panel shows the far-UV luminosity function, ${ }^{20}$ assuing $\Omega_{0}=0.3, \Omega_{\Lambda}=0.7, \mathrm{~h}=1$. The typical LBG is extinguished by 1.5-2 magnitudes at rest-frame $1600 \AA \AA^{20,11}$ (Right) The inferred star formation history of the universe to $z \sim 5$, where the two highest redshift points are from extensive photometric and spectroscopic LBG samples at $\sim 3$ and $z \sim 4.1{ }^{11}$ All of the points have been corrected for extinction assuming the distribution shown in the left-hand panel.

forward in time, most of the stars that are seen forming at $z \sim 3-4$ would today be incorporated into galaxies living in the richest environments, like clusters and rich groups. ${ }^{14,19}$

The large spectroscopic samples, made more useful because the selection method produces samples that are nearly volume-limited, have also allowed accurate (UV) luminosity distributions to be determined, as shown in Figure 3. They have also allowed for careful examination of the distribution of continuum spectral energy distributions, leading to inferred distribution functions for extinction and reddening (also illustrated in Figure 3). These numbers are essential for estimating the amount of energy produced by star formation that is not accounted for in the rest-frame UV measurements. Careful analysis of the distributions shows that the LBGs are likely to be the major contributors to the far-IR/sub-mm background over the redshift range $1 \lesssim z \lesssim 5 .^{20}$ Finally, it has become possible to address the global star formation history of the universe by combining the large ground-based samples of very high redshift galaxies with the results of redshift surveys to $z \sim 1$, as in Figure 3b. Curiously, while initial results from analysis of small photometric LBG samples from the HDF-N indicated a rather rapid diminution of the global star formation density for $z>2,{ }^{21}$ more recent consideration of the large samples based on ground based imaging and spectroscopy ${ }^{11}$ indicates that the rate of star formation remains high to at least $z \sim 4.5$. This makes an important point: the large fields covering large volumes that are currently accessible only using ground-based facilities are probably necessary for any accurate statistics on the high redshift universe. Another point worth noting from the bottom panel of Figure $3 \mathrm{~b}$ is that the redshift range corresponding to the most important epoch for the global production of stars (and therefore presumably it is the most important epoch for galaxy assembly) falls in the redshift range $1 \lesssim z \lesssim 4$. Happily, as discussed both above and below, this range is highly accessible to current and future instrumentation of $8-10 \mathrm{~m}$ telescopes.

\subsection{Galaxies at Very High Redshifts $(z>5)$}

While it has become possible to undertake extensive surveys of the universe over the redshift range $1 \lesssim z \lesssim 4$, as summarized above, there has also been intensive exploratory work on pushing the telescopes to their limit and extending the redshift range beyond $z \sim 4.5$ into the so-called "dark ages". While NGST has been designed specifically to address the universe of galaxies at these extremely high redshifts, there is still nearly a decade before NGST is available, providing a substantial window of time to begin to open up the "dark ages" using the groundbased telescopes. 
Most of the work that has been done so far at the highest redshifts has been follow-up of color-selected object discovered using $H S T^{22,23}$ or has been focused on the use of Lyman $\alpha$ emission as the principal means for discovery either using narrow-band imaging ${ }^{24}$ or "controlled serendipity". ${ }^{25}$ The pursuit of the highest redshifts is described in much more detail in McMahon's contribution to this volume, and also in reference $26 .^{26}$

The observations at redshifts beyond $z \sim 5$ are extremely difficult even with $8-10 \mathrm{~m}$ telescopes due to the diminishing brightness of the objects coupled with the increasing amplitude and complexity of the sky background; some improvement may be realized with new CCD detectors now under development with extremely high QE out to $1 \mu \mathrm{m}$ with essentially no fringing. However, it may not ever be possible to achieve the same kinds of statistical results as at $z<4$, where everything is much easier.

\subsection{Supporting Roles: Follow-up of Mid-IR, Sub-mm, Radio, X-ray, and Gamma Ray Sources}

State-of-the-art imaging spectrographs, operating in the optical and near-IR, are the workhorse instruments of almost any high redshift galaxy program, no matter what techniques or wavelengths have been used to identify the sources. Large ground-based optical/IR telescopes have already been used extensively for follow-up of the newly-discovered $850 \mu \mathrm{m}$ sources and the apparently-related micro-Jy radio sources. ${ }^{27,28}$ The new optical/IR facilities have also been used for follow-up of extremely deep ROSAT data ${ }^{29}$; with the launch of CHANDRA and XMM, it is anticipated that follow-up of the much fainter and more numerous $\mathrm{X}$-ray sources from the high resolution imaging instruments will be a major industry over the next decade. Optical/IR facilities have also been responsible for much of the progress made in following up high energy transients, including the first confirmation of the extragalactic nature of Gamma Ray Bursts, ${ }^{30}$ and many subsequent high redshift GRB host galaxy spectroscopic identifications; Kulkarni (this volume) describes these developments in detail. The imminent launch of SIRTF will provide a wealth of opportunity for the new ground-based telescopes for follow-up of mid-IR sources. Until the advent of ALMA and NGST late in this decade, optical/IR spectroscopy from the ground will provide the greatest sensitivity, and sometimes the only hope, for determining the nature of distant astronomical sources discovered using both satellites and ground-based long-wavelength techniques.

\subsection{The Advent of Sensitive Near-IR Spectroscopy}

While near-IR spectroscopic observations of high redshift galaxies have been possible using $4 \mathrm{~m}$-class telescopes (given significant tenacity and patience! ${ }^{31}$ ), it is only in the last 6 months, most notably with NIRSPEC and ISAAC that it becomes possible to make substantial headway on significant samples. Some preliminary results of our own observational programs using these instruments have been presented elsewhere. ${ }^{32}$

The motivations for observing distant galaxies in the 1-2.5 $\mu \mathrm{m}$ range are several: most obvious is that galaxies without current star formation lying at redshifts beyond $z \sim 1$ simply have little or no flux in the optical window (0.4-1.0 microns); heavily reddened star forming galaxies at $z \gtrsim 1$ may also be more easily observed in the near-IR (depending on the redshift and the degree of UV extinction). There is also the notorious problem of securing redshifts for star-forming galaxies in the range $1.3 \lesssim z \lesssim 2.5$ because the spectral features generally used at $z \leq 1$ for securing redshifts are no longer accessible in the optical window. This redshift range takes on added importance since it now appears that it encompasses a large fraction of the most important epoch for star formation in the universe (figure 3).

In many respects, the greatest utility of the near-IR spectrographs is in their power for diagnostic spectroscopy of previously-discovered high redshift galaxies, rather than for blind redshift identifications (to be discussed below). The two most significant astrophysical quantities that can be measured more effectively in the near-IR than in the optical are galaxy kinematics (line widths, and possibly rotation curves ${ }^{31}$ ) and estimates of chemical abundances. ${ }^{33,32}$ As shown in the example in Figure 4, one can perform continuum spectroscopy in the optical that can reveal a wealth of UV absorption lines from both stars and the galaxy interstellar medium, whereas in the near-IR, continuum spectroscopy is essentially hopeless for normal star forming galaxies, of which even the brightest have $K_{A B} \sim 22$ at $z \sim 3$. However, it may well be that the information content of the near-IR spectrum is higher. In the rest-frame far-UV, the interstellar lines are broadened by turbulent motions and are significantly blue-shifted relative to the galaxy's systemic redshift; the stellar lines are difficult to measure in all but the very best spectra. ${ }^{34}$ Lyman $\alpha$ is severely affected by radiative transfer effects, and is not seen in emission in more than $50 \%$ of the star forming galaxies at $z \sim 33^{35}$ With subsets of the familiar optical nebular lines accessible in the near-IR for redshifts $1 \lesssim z \lesssim 4.5$ $(z=2.2-2.5$ is a "magic" redshift for near-IR spectroscopy as it places [OII] $\lambda 3727$ in the J band, [OIII] and H $\beta$ in 

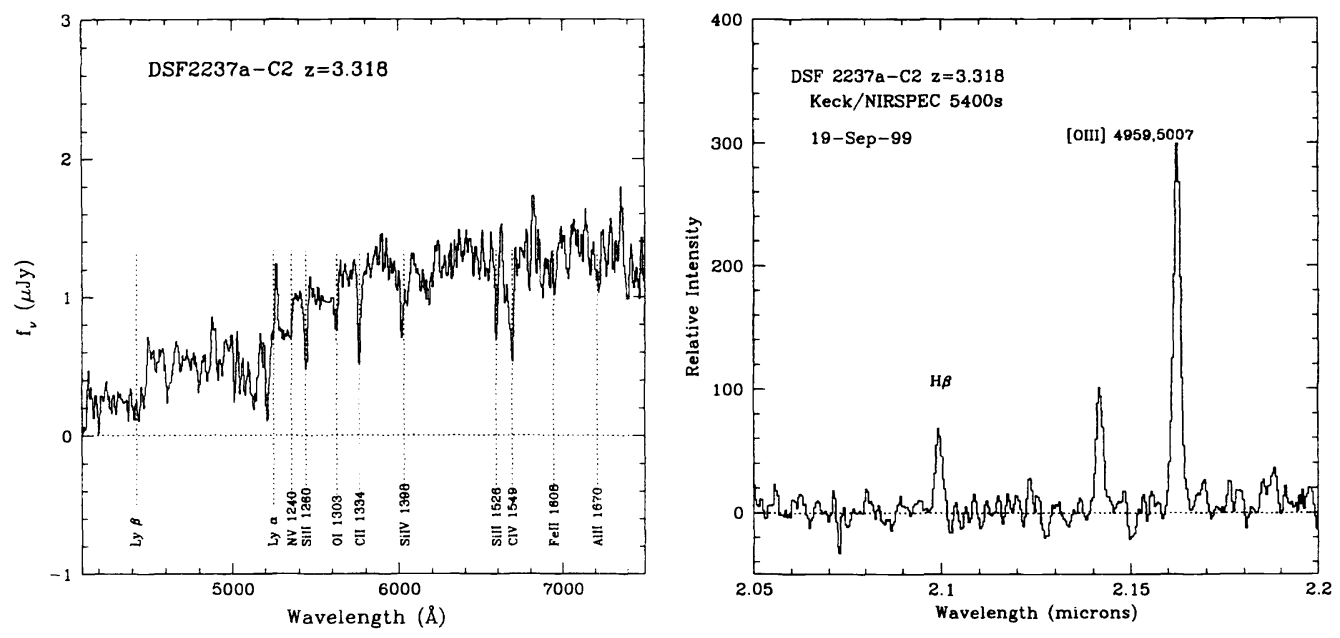

Figure 4. Examples of optical (left) and near-IR (right) spectra of a $z \sim 3$ galaxy. The optical spectrum was obtained with LRIS, with a total integration time of 5400s; the near-IR spectrum was obtained with a similar integration time, with a priori knowledge of the redshift, using Keck/NIRSPEC soon after commissioning in the fall of 1999 .

the $\mathrm{H}$ band, and $\mathrm{H} \alpha+[\mathrm{NII}]$ in the $\mathrm{K}$ band atmospheric windows), one can obtain both dynamical line widths (subject to interpretation regarding the extent to which they can be trusted to measure the most interesting quantity- mass) and astrophysically meaningful line intensity ratios, notably the ratio $R_{23} \equiv([O I I I]+[O I I]) / \mathrm{H} \beta$, which has been calibrated locally ${ }^{33}$ as a measure of the average abundance in the integrated spectrum of HII regions. However, because of the gaps between the atmospheric windows and the ubiquitous $\mathrm{OH}$ sky lines in the near-IR, even emission line spectroscopy is far from easy and in our experience the redshifts must be chosen very carefully to successfully measure multiple emission lines.

\section{LOOKING TOWARD THE FUTURE}

While there are almost infinite possibilities for work on high redshift galaxies using 8-10m telescopes in the future, I outline some of my favorite applications below. These projects are all survey-oriented, and neglect the power that can brought to bear on high redshift studies using such techniques as adaptive optics, integral field spectroscopy, and high dispersion studies of individual (bright) objects. They also do not do adequate service to the continued pursuit of the very highest redshifts. This reflects only my own personal scientific tastes, and the general feeling that there is much to be learned with real statistically robust samples at the more accessible redshifts $(1 \lesssim z \lesssim 4)$. These redshifts also allow for spectroscopic work at both optical and near-IR wavelengths

\subsection{Opening the $z=1.5-2.5$ Universe}

A large part of the motivation for the development of multi-plexing near-IR spectrographs for 8-10m telescopes is driven by the desire to follow the evolution of galaxies into this very difficult redshift range. Most efforts to address this range of redshifts are looking toward the near-IR because familiar rest-frame optical emission lines can be recovered; however, there are many advantages in working in the near-UV and blue as an alternative. First, the terrestrial background is from 50 to 1000 times darker at 0.35 microns than at $>1 \mu \mathrm{m}$, and the near-UV/blue background is relatively featureless continuum rather than the combination of bright continuum and complex molecular absorption and emission bands in the near-IR that makes sky subtraction especially prone to systematics and that limits sensitivity. Progress in detectors, optics, and optical coatings are making spectroscopic throughput in the near-UV as high as in the visual and near-IR.

I strongly suspect that multi-plexed IR spectroscopy will prove relatively inefficient as a means of measuring redshifts for faint galaxies; even the brightest Lyman break galaxies $\left(\mathcal{R}_{\mathrm{AB}} \sim 23-24\right)$ require great care in selection 

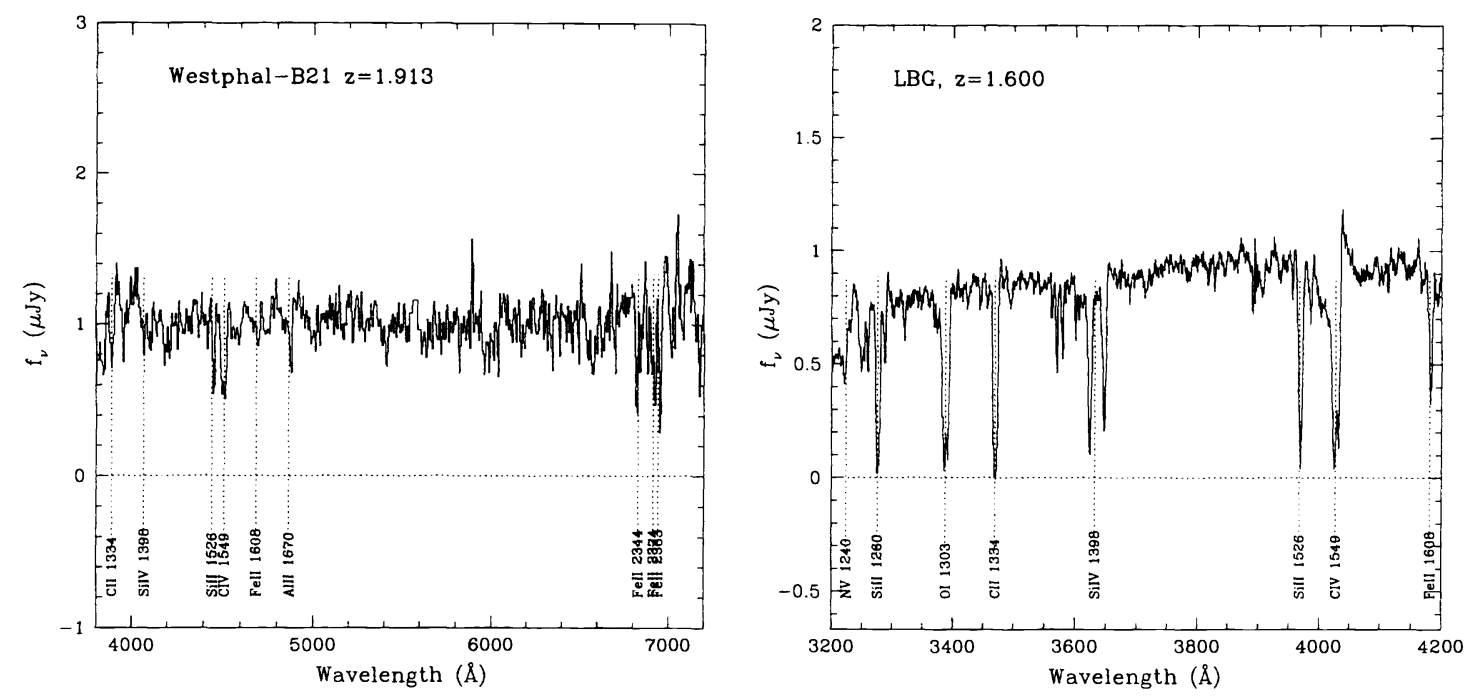

Figure 5. (Left) An example of $z \sim 2$ spectrum obtained with Keck/LRIS. The spectrograph efficiency currently plummets at $4000 \AA$, so that many of the strongest UV lines are inaccessible for lower redshift objects. The right panel illustrates the appearance of the spectrum of a LBG placed at $z=1.6$, observed with the wavelength range and resolution achievable with LRIS-B.

(including a priori knowledge of the redshifts) and long integrations to yield successful results for near-IR spectroscopy, as mentioned above. On the other hand, experience has shown us that absorption line redshifts obtained from lines appearing in the observed-frame blue and visual are highly effective to $B \sim 25.5$ using LRIS for $z \sim 3$ galaxies. Experiments conducted in the course of work with LRIS suggest that extensive headway can be made into the "spectroscopic desert" at $z \sim 1.3-2.5$ not only by pushing multiplexed spectroscopy into the near-IR to follow the familiar nebular rest-frame optical lines to $z>1$, but also by using the same set of spectroscopic features that have proved so efficient in identifying $z \sim 3$ galaxies as they enter the ground-based window from the UV. These spectroscopic features, due to the ubiquitous interstellar absorption lines of resonance transitions of ions like OI, SiII, CII, CIV, SiIV, FeII, and MgII, and the strong high ionization stellar features produced by massive stars (e.g., CIV, SiIV, NV) are found throughout the vacuum UV from Lyman $\alpha$ at $1216 \AA$ to Mg II at $\lambda \lambda 2796,2803$, but are especially strong in the 1200-1800 $\AA$ region in the rest frame. The typical strength of the strongest lines are $\sim 5 \AA$ in the rest frame, and the sheer number of transitions accessible in the UV has been directly responsible for the success of spectroscopic identifications of nearly $1000 z \sim 2.5-4.5$ galaxies (figure 1) despite the fact that most of the galaxies show no strong emission lines in the observed-frame optical. ${ }^{35}$ An example of a spectrum of a $z \sim 2$ galaxy, obtained using LRIS in exploratory manner in the course of the $z \sim 3$ LBG survey, is shown in figure 5, along with an illustration of how the near-UV could be very effective for even the most difficult redshifts.

The success of such spectroscopic identifications so far rapidly decreases at $z \lesssim 2.4$ because the efficiency of LRIS, and other imaging spectrographs on 8-m class telescopes, falls off sharply shortward of $4000 \AA$ because of design trade-offs taken to optimize performance in the visual and red spectral regions. However, it is clear that a new opportunity will exist for exploring the heretofore barely-touched $z \sim 1.3-2.5$ regime by making use of wavelengths all the way down the atmospheric cutoff near $3100 \AA$.

This opportunity is going to be exploited by a new instrument to to be installed on the Keck I telescope that involves adding a UV/Blue optimized channel to the existing LRIS. This new facility instrument (LRIS-B) is expected to be shipped to Hawaii in the spring of 2000, and to be commissioned during the late summer of 2000. LRIS-B will offer a capability in the 3000-4000 $\AA$ region that will be unique in the world, at an observatory where the UV atmospheric opacity is significantly lower than at any other developed optical/IR site. LRIS in its new incarnation will allow high throughput observations from the atmospheric cutoff to 1 micron, simultaneously (because the blue side is optimized for $3000-5000 \AA$ and the red side for $5000-10000 \AA$; the two beams observe the same field, with the wavelength ranges divided by a dichroic beam splitter). Because of this unparalleled spectral coverage in multi-slit 
mode, LRIS will be a particularly effective "redshift machine" for working on the very blue objects which dominate the number counts of faint galaxies, where photometric redshifts are least constraining, and where the least is known about the statistical properties of the galaxy populations.

\subsection{The "Cosmic Web" at High Redshift}

Historically, there has been somewhat of a "disconnect" between the information provided on gas in galaxies and the IGM at high redshift by QSO absorption lines studies, and that provided by direct studies of galaxies using standard faint galaxy techniques. The main reason for this is that it has generally been difficult to obtain information on the same cosmic epochs using the two different observational techniques; however, advances in the precision and detail with which one can study gas at high redshifts via QSO absorption line techniques (see Rauch's contribution to this volume) with the advent of echelle spectrographs on 8-10m class telescopes have been paralleled by advances in the redshifts at which statistical properties of galaxies can be studied. It is possible to join these capabilities in a focused observational effort to tie together galaxies and their large scale distribution at $2.5 \lesssim z \lesssim 3.5$ (for example) with observations of the Lyman alpha forest in the same cosmological volumes. The aim is to probe both the collapsed and uncollapsed baryonic components of the universe at a particular cosmic epoch where the two can be concurrently studied in a highly efficient manner using observational techniques that are now well-established.

We are currently working on a project to map the galaxy distribution in $\sim 10^{\prime}$ fields surrounding suitably chosen QSOs for which we already have (or soon will have) very high quality Keck/HIRES spectra. Examples of the kinds of data to be used are given in Figures 6 and 7. The objective is to use the Lyman break technique to identify galaxies and their large-scale distribution within a redshift interval $2.5 \lesssim z \lesssim 3.5$ over which both the Lyman $\alpha$ forest and the metal lines (most notably C IV) are observable in the QSO spectrum. By choosing the QSO redshift to be just "behind" the redshift interval over which the $(z \sim 3)$ Lyman break galaxy identification is most sensitive, there is an optimally large interval over which a comparison can be made between the galaxies and the absorption line systems. This type of observation seems particularly timely now that the state of $\mathrm{N}$-body +hydrodynamical simulations is such that the same models can treat both galaxy formation and the diffuse gas in the Lyman $\alpha$ forest. ${ }^{36,37}$

In the context of the current numerical models, ${ }^{38,39}$ the Lyman $\alpha$ forest arises from diffuse gas close to the mean density of the universe that is a largely unbiased tracer of the mass distribution; on the contrary, we already know that the galaxies must be highly biased tracers of the mass in the context of any hierarchical galaxy formation paradigm ${ }^{14-16}$ The observations probing the same volumes and sensitive to a huge dynamic range in baryonic density will provide, for the first time, a means to directly cross- correlate fluctuations in the low column density gas (a proxy for the mass fluctuations) and the galaxies at very high redshifts.

It is difficult to predict a priori what will be found in this type of study; it will be very interesting to compare the properties of the Lyman $\alpha$ forest within and outside of regions of galaxy over-densities, including the metal content which can be so sensitively probed using high $\mathrm{S} / \mathrm{N}$ echelle spectra. ${ }^{40}$ In addition to the prospects for measuring the structures as discussed above, there are many possible astrophysical effects that might be measurable, from metallicity enhancements of the Lyman $\alpha$ forest systems in regions of overall higher galaxy density, to ionization effects produced by the proximity of many luminous star forming galaxies. For example, it is possible that the proposed observations could settle the question of whether the metal abundances in the Lyman $\alpha$ forest are uniform and "pre-galactic" in origin, or whether only the gas that lies in the vicinity of galaxy concentrations has been polluted by metals from star formation. This program is in many ways exploratory, and we hope will indicate whether this type of study will prove fruitful (on a much larger scale) in the future.

\subsection{Very Large Galaxy Surveys at High Redshift}

A very intriguing possibility for the future is to undertake surveys of high-redshift galaxies that cover co-moving volumes comparable to the most ambitious surveys of the local universe. It now seems possible that such programs can be accomplished, particularly by applying the new-found ability to do essentially volume-limited surveys of galaxies at prescribed cosmic epochs using sensible photometric pre-selection. There are a few considerations that show that photometric pre-selection is probably a desirable ingredient: just as an illustrative example, suppose one were simply to impose a selection criterion $I_{A B} \leq 24.5$ and that the survey size is intended to have 50,000 redshifts (i.e., similar to the planned deep VIRMOS survey). Given what we now know from color-selected surveys, ${ }^{11}$ the full sample would contain approximately 1000 galaxies in the redshift range $2.7 \lesssim z \lesssim 3.4$ and approximately 250 galaxies in the redshift range $3.8 \lesssim z \lesssim 4.5$. These numbers are not appreciably different from the existing samples at these redshifts, and the sampling at such bright apparent magnitudes will be very dilute, making evaluation of 

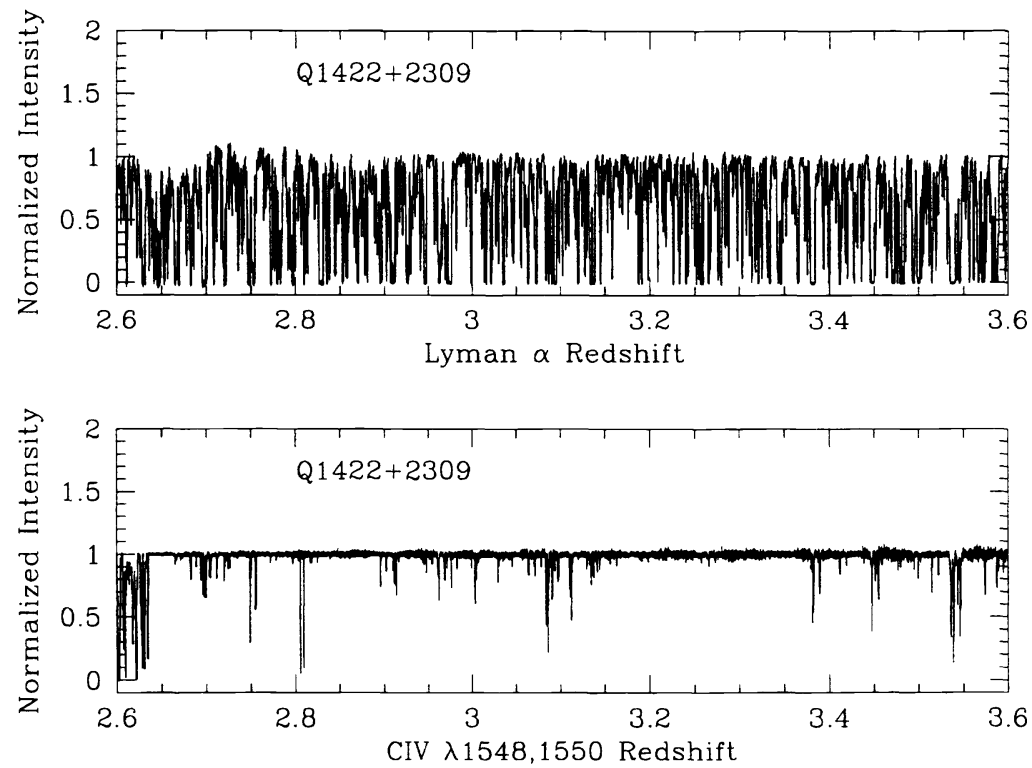

Figure 6. The HIRES spectrum of Q1422+2309 (courtesy W. Sargent), illustrating the kind of data that can be compared with the galaxy distribution obtained from a combination of deep imaging and LRIS spectroscopy. The top panel shows the Lyman $\alpha$ forest over the redshift range of interest; the bottom panel shows the corresponding C IV doublets. These plots are purely illustrative; it is not possible to convey the quantity of information contained in the spectra seen at their full resolution.
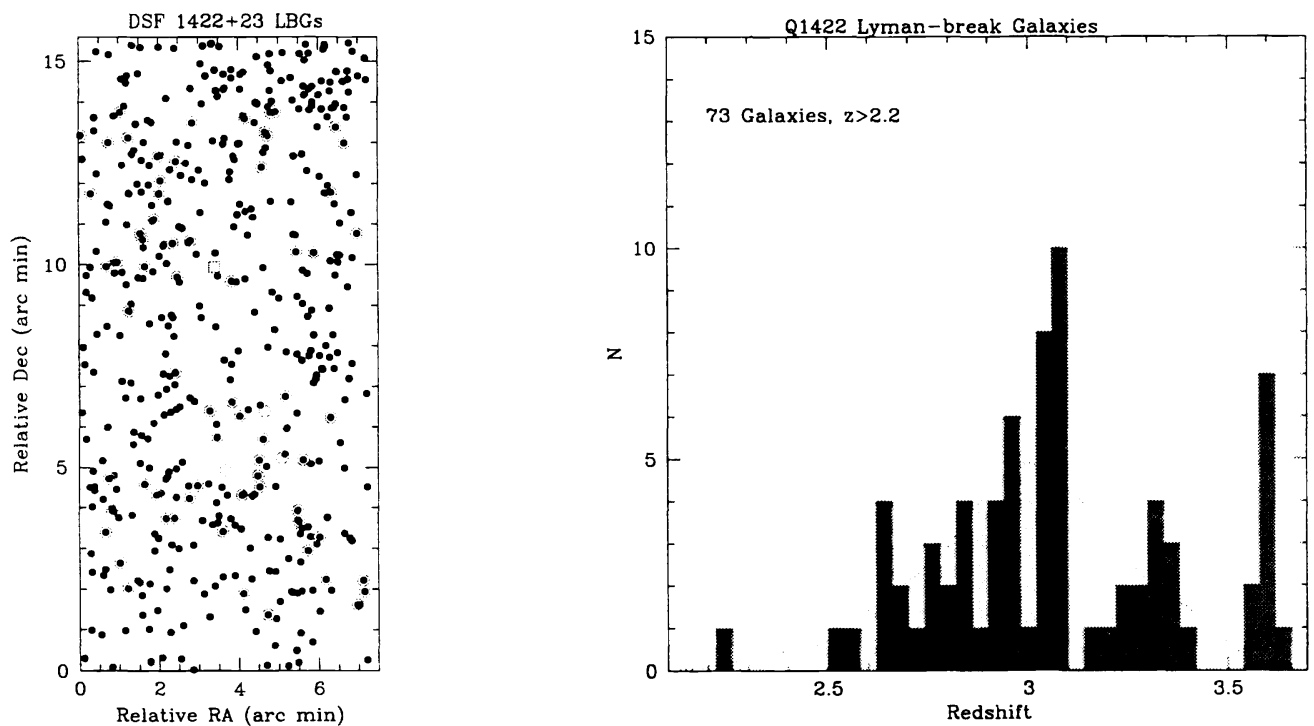

Figure 7. (Left) Map of photometrically selected $2.6 \leq z \leq 3.6$ galaxies on the plane of the sky in the field of the Q1422+2309 $\left(z_{e m}=3.62\right)$; circled objects have spectroscopic redshifts at the time of this writing. (Right) Current redshift histogram in the Q1422+2309 field, together with the overall selection function (light histogram) of the color selection criteria. 
large scale structure considerably more difficult. On the other hand, a straight apparent magnitude-limited survey in some ways allows for a more straightforward interpretation of the resulting sample and certainly is a better way to go at $z \lesssim 1$ to ensure that one is seeing everything that is there, regardless of star formation history. The bottom line is that no single survey is going to be able to address all interesting problems in galaxy evolution simultaneously.

For this reason, the most effective surveys in the future will be relatively targeted ones. An example of a targeted redshift survey is the DEEP survey, ${ }^{41}$ which will be conducted using the DEIMOS spectrograph on Keck II beginning in the early part of 2001 . In this case, photometric pre-selection will be used to exclude galaxies with $z \lesssim 0.7$ and the bulk of the survey effective volume is likely to lie in the range $0.7 \leq z \leq 1.4$. By constraining the targeted redshift range, DEEP will be able to use significantly higher spectral resolution (with its accompanying penalty in spectral coverage) and will therefore obtain very accurate velocities as well as spectra of high enough quality to use for measuring the internal motions of the galaxies. Both of these benefits will allow the survey to address issues of galaxy mass and the relation of the galaxy distribution to the overall mass distribution. This is another important point: merely collecting huge numbers of redshifts is probably not going to solve some of the most interesting scientific questions, and is another reason why catch-all surveys may not be the most effective way to go in the future.

The ability to obtain very deep, high quality images over large $\left(\sim 30^{\prime}\right)$ fields (mostly on 4m-class telescopes), together with the next generation of imaging spectrographs on $8 \mathrm{~m}$-class telescopes which can cover these fields efficiently, makes feasible truly large-scale surveys of the distant universe. My own choice along these lines would be to target the $z \sim 2.5-3.5$ universe in particular (for the practical reasons outlined above). Survey fields of $\sim 1-2$ degrees in transverse extent would cover co-moving scales larger than the largest structures in the present-day universe (at $z \sim 3$, one degree covers a co-moving transverse dimension of $75 h^{-1} \mathrm{Mpc}$ for $\Omega_{0}=0.3, \Omega_{\Lambda}=0.7$ ). We have found that, with photometric pre-selection, a high degree of spectroscopic completeness is possible to $\mathcal{R}=25.5$ $\left(I_{\mathrm{AB}} \approx 25.3\right)$. To this apparent magnitude level, within this particular redshift range, there are $\sim 6000 \mathrm{LBGs}$ per square degree. Whereas preliminary measurements of clustering at high redshift have already been made, surveys with several independent fields covering such large areas would allow one to actually map the topology of large scale structure at $z \sim 3$ in addition to improving several-fold the precision with which the clustering is measured. And, such a survey could be completed with a relatively modest investment of $8 \mathrm{~m}-10 \mathrm{~m}$ telescope time ( $\sim 30$ clear nights).

Having said all of this, it is difficult to choose the most interesting direction to go with galaxies in the high redshift universe; we have only scratched the surface of the potential of our new telescopes and instruments. The high redshift universe is wide open, and the epoch of galaxy formation is fully accessible to our exploration. It is going to be a very exciting decade.

\section{ACKNOWLEDGMENTS}

I would like to thank my collaborators, Kurt Adelberger, Max Pettini, Alice Shapley, Mark Dickinson, and Mauro Giavalisco for their contributions to much of the work discussed here. Of course, the work would not be possible without the generous gift of the W.M. Keck Foundation which allowed construction of the Keck Observatory, and the instrument and telescope builders whose work enables the science. Most of the work described above has been supported by the U.S. National Science Foundation through grant AST95-96229, and by the David and Lucile Packard Foundation. Finally, I would like to acknowledge financial support from the European Southern Observatory that allowed me to attend this fascinating meeting.

\section{REFERENCES}

1. J.B. Oke et al., "The Low Resolution Imaging Spectrometer for the Keck Telescope", P.A.S.P., 107, 375-385, 1995.

2. I.S. McLean et. al., "The Design and Development of NIRSPEC: A Near-Infrared Echelle Spectrograph for the Keck II Telescope", SPIE, 3354, 566-578, 1998.

3. N.P. Vogt, D.A. Forbes, A.C. Phillips, C. Gronwall, S.M. Faber, G.D. Illingworth, \& D.C. Koo , “Optical Rotation Curves of Distant Field Galaxies: Keck Results at Redshifts to $z \sim 1$ ", Ap.J., 465, L15-L18, 1996.

4. S.J. Lilly, L. Tresse, F. Hammer, D. Crampton, \& O. Le Févre, "The Canada-France Redshift Survey.VI. Evolution of the Galaxy Luminosity Function to $z \sim 1$ ", Ap.J., 455, L108-111,1995

5. J.G. Cohen, D.W. Hogg, M.A. Pahre, \& R.D. Blandford, "Strong Redshift Clustering of Distant Galaxies", Ap.J., 462, L9-L12, 1996 
6. L.L. Cowie, A. Songaila, E.M. Hu, \& J.G. Cohen, "New Insight on Galaxy Formation and Evolution from Keck Spectroscopy of the Hawaii Deep Fields", A.J., 112, 112-125, 1996

7. C.C. Steidel \& K.L. Adelberger, "High Redshift Galaxies: What Good Are They?", in Photometric Redshifts and the High Redshift Universe, R. Weymann, L. Storrie-Lombardi,eds., pp. 227-243, Astr. Society of the Pacific, 1999

8. C.C. Steidel, M. Pettini, \& D. Hamilton, "Lyman Limit Imaging of High-Redshift Galaxies.3. New Observations of 4 QSO Fields", A.J., 110, 2519-2536, 1995

9. C.C. Steidel, M. Giavalisco, M. Pettini, M. Dickinson, \& K.L. Adelberger, "Spectroscopic Confirmation of a Population of Normal Star-Forming Galaxies at Redshifts $z>3$ ", Ap.J., 462, L17-L21, 1996

10. J.D. Lowenthal, et al., "Keck Spectroscopy of Redshift $z \sim 3$ Galaxies in the Hubble Deep Field", Ap.J., 481, 673-686, 1997

11. C.C. Steidel, K.L. Adelberger, M. Giavalisco, M. Dickinson, \& M. Pettini, "Lyman Break Galaxies at $z \gtrsim 4$ and the Evolution of the UV Luminosity Density at High Redshift", Ap.J., 519, 1-17, 1999.

12. K.L. Adelberger, "Star Formation and Structure Formation at Redshifts $1 \leq z \leq 4$ ", in Clustering at High Redshift, A. Mazure \& O. Le Févre, eds., in press, 2000.

13. K.L. Adelberger, K.L., et. al., in preparation, 2000.

14. C.C. Steidel, K.L. Adelberger, M. Dickinson, M. Giavalisco, M. Pettini, \& M. Kellogg, "A Large Structure of Galaxies at $z \sim 3$ and its Cosmological Implications", Ap.J., 492, 428-438, 1998.

15. K.L. Adelberger, C.C. Steidel, M. Giavalisco, M. Dickinson, \& M. Pettini, "A Counts-in-Cells Analysis of Lyman Break Galaxies at $z \sim 3 "$, Ap.J., 505, 18-24, 1998.

16. C.M. Baugh, S. Cole, C.S. Frenk, \& C.G. Lacey, "The Epoch of Galaxy Formation", Ap.J., 498, 504-520, 1998.

17. H. Mo, S. Mao, S., \& S.D.M. White, "The Structure and Clustering of Lyman Break Galaxies", MNRAS, 304, 175-184, 1999.

18. R.H. Wechsler, M.A.K. Gross, J.R. Primack, G.R. Blumenthal, \& A. Dekel, "Implications of Spikes in the Redshift Distribution of $z \sim 3$ Galaxies", Ap.J., 506, 19-32, 1998.

19. F. Governato, B. Moore, C.S. Frenk, S. Cole, C.G. Lacey, \& J. Stadel, "The Seeds of Rich Galaxy Clusters in the Universe", Nature, 392, 359-361, 1998.

20. K.L. Adelberger \& C.C. Steidel, "Constraints on Dusty Star Formation at High Redshift from Ultraviolet, Far-IR, and Radio Surveys", Ap.J., submitted, 2000.

21. P. Madau, H.C. Ferguson, M. Dickinson, M. Giavalisco, C.C. Steidel, \& A.S. Fruchter, "High Redshift Galaxies in the Hubble Deep Field: Color Selection and Star Formation History to $z \sim 4$ ", MNRAS, 283, 1388-1404, 1996.

22. R.J. Weymann, D. Stern, A. Bunker, H. Spinrad, F.H. Chaffee, R. Thompson, \& L.J. Storrie-Lombardi, "Keck Spectroscopy and NICMOS Photometry of a Redshift $z=5.60$ Galaxy", Ap.J., 505, L95-L98, 1998

23. H. Spinrad, H., "A $z=5.34$ Galaxy Pair in the Hubble Deep Field", A.J., 116, 2617-2623, 1998.

24. E.M. Hu, L.L. Cowie, \& R.G. McMahon, "An Extremely Luminous Galaxy at $z=5.74$ ", Ap.J., 522, L9-L12, 1999.

25. A. Dey, H. Spinrad, D. Stern, J.R. Graham, \& F.H. Chaffee, "A Galaxy at $z=5.34$ ", Ap.J., 498, L93-L96, 1998.

26. D. Stern \& H. Spinrad, "Search Techniques for Distant Galaxies", PASP, 111, 1475-1502, 1999.

27. R. Ivison, I. Smail, J.-F. Le Borgne, A. Blain, J.-P. Kneib, J. Bezecourt, T.H. Kerr, \& J.K. Davies, "A HyperLuminous Galaxy at $z=2.8$ Found in a Deep Sub-millimeter Survey", MNRAS, 298, 583-593, 1998.

28. A.J. Barger, L.L. Cowie, \& E.A. Richards, "Mapping the Evolution of High Redshift Dusty Galaxies with Sub-millimeter Observations of a Radio-Selected Sample", A.J., in press, 2000.

29.

30. M.R. Metzger, S. Djorgovski, S. Kulkarni, C.C. Steidel, K.L. Adelberger, D.A. Frail, E. Costa, \& F. Frontera, "Spectral Constraints on the Redshift of the Optical Counterpart to the Gamma Ray Burst of 8 May 1997", Nature, 387, 879-881, 1997.

31. M. Pettini, M. Kellogg, C.C. Steidel, M. Dickinson, K.L. Adelberger, \& M. Giavalisco, "Infrared Observations of Nebular Emission Lines from Galaxies at $z \approx 3 "$, Ap.J., 508, 539-550, 1998.

32. M. Pettini, "The First Galaxies: Clues from Element Abundances", Phil. Trans. R.S., in press, 2000. 
33. H.A. Kobulnicky, R.C. Kennicutt, \& J.L. Pizagno, "On Measuring Nebular Chemical Abundances in Distant Galaxies Using Global Emission-Line Spectra", Ap.J., 514, 544-557, 1999.

34. M. Pettini, C.C. Steidel, K.L. Adelberger, M. Dickinson, \& M. Giavalisco, "The Ultraviolet Spectrum of MS 1512-cB58: An Insight into Lyman-Break Galaxies", Ap.J., 528, 96-107, 2000.

35. C.C. Steidel, K.L. Adelberger, A.E. Shapley, M. Pettini, M. Dickinson, \& M. Giavalisco, "Lyman $\alpha$ Imaging of a Proto-Cluster Region at $z=3.09 ", A p . J .$, in press, 2000.

36. N. Katz, L. Hernquist, \& D.H. Weinberg, "The Clustering of High-Redshift Galaxies in the Cold Dark Matter Scenario", Ap.J., 523,463-479, 1999.

37. R. Cen \& J.P. Ostriker, "Cosmic Chemical Evolution", A p.J., 519, L109-L113, 1999.

38. L. Hui \& N.Y. Gnedin, "Equation of State of the Photo-ionized Intergalactic Medium", MNRAS, 292, 27-42, 1997.

39. Croft, R.A.C., Weinberg, D.H., Katz, N., \& Hernquist, L., "Recovery of the Power Spectrum of Mass Fluctuations from Observations of the Lyman Alpha Forest", Ap.J., 495, 44-62, 1998.

40. L.L. Cowie, \& A. Songaila, "Heavy-Element Enrichment in Low-Density Regions of the Intergalactic Medium", Nature, 394, 33-36, 1998.

41. M. Davis \& S.M. Faber, "The DEIMOS Spectrograph and a Planned DEEP Redshift Survey", in Wide Field Surveys in Cosmology, S. Colombi, Y. Mellier, B. Raban, eds., pp. 333-338, Editions Frontieres, Paris, 1998. 